\title{
Subarachnoid small vein occlusion due to inflammatory fibrosis-a possible mechanism for cerebellar infarction in cryptococcal meningoencephalitis: a case report
}

Yoshiteru Shimoda ${ }^{1,2^{*}}$, Satoru Ohtomo ${ }^{1}$, Hiroaki Arai ${ }^{1}$, Takashi Ohtoh ${ }^{3}$ and Teiji Tominaga ${ }^{2}$

\begin{abstract}
Background: Cryptococcal meningoencephalitis (CM) causes cerebral infarction, typically, lacunar infarction in the basal ganglia. However, massive cerebral infarction leading to death is rare and its pathophysiology is unclear. We report a case of CM causing massive cerebellar infarction, which led to cerebral herniation and death.

Case presentation: A 56-year-old man who suffered from dizziness and gait disturbance for one month was admitted to our hospital and subsequently diagnosed with a cerebellar infarction. He had a past medical history of hepatitis type B virus infection and hepatic failure. Although the findings on magnetic resonance imaging (MRI) imitated an arterial infarction of the posterior inferior cerebellar artery, an accompanying irregular peripheral edema was observed. The ischemic lesion progressed, subsequently exerting a mass effect and leading to impaired consciousness. External and internal decompression surgeries were performed. Cryptococcus neoformans was confirmed in the surgical specimen, and the patient was diagnosed with CM. In addition, venule congestion in the parenchyma was observed with extensive fibrosis and compressed veins in the subarachnoid space. The patient died 26 days after admission. Autopsy revealed that pathological changes were localized in the cerebellum.

Conclusion: C. neoformans can induce extensive fibrosis of the subarachnoid space, which may compress small veins mechanically inducing venule congestion and massive cerebral infarction. In such cases, the clinical course can be severe and even rapidly fatal. An atypical pattern of infarction on MRI should alert clinicians to the possibility of $C$. neoformans infection.
\end{abstract}

Keywords: Cryptococcal infection, Vein occlusion, Magnetic resonance imaging, Autopsy

\section{Background}

Cryptococcal meningoencephalitis (CM) caused by Cryptococcus neoformans infection is the most common fungal infection of the central nervous system $[1,2]$. Throughout its clinical course, C. neoformans induces small cerebral infarctions such as lacunar infarctions primarily in the basal ganglia [3-8]; however, massive brain

\footnotetext{
*Correspondence: yositeru8_8_8simoda@yahoo.co.jp

'Department of Neurosurgery, South Miyagi Medical Center, 38-1 Azanishi,

Ogawara-machi, Shibata-gun, Miyagi 989-1253, Japan

${ }^{2}$ Department of Neurosurgery, Tohoku University Graduate School of

Medicine, Sendai, Japan

Full list of author information is available at the end of the article
}

infarction causing cerebral herniation has not been well described. We present a case of a patient with $\mathrm{CM}$ who presented with an atypical cerebellar infarction, which ultimately caused fatal cerebral herniation. Pathological and radiological findings revealed possible underlying pathogenesis.

\section{Case presentation}

A 56-year-old man presented to our department with headache, vomiting, and gait disturbance (for 1 month). He had a past medical history of hepatitis type B virus infection and hepatic failure. He had been medically 
treated for hypertension and hepatitis for the previous 4 years. On admission, he had an impaired consciousness [Glasgow coma scale (GCS), 14]. Cerebellar ataxia and gait disturbance were evident. Diffusion weighted imaging (DWI) demonstrated multiple cerebellar infarctions at several intensities with perilesional edema of the left cerebellar hemisphere (Fig. 1a, b). Brain magnetic resonance imaging (MRI) did not reveal any prominent meningeal gadolinium enhancement or nodule (Fig. 1c). MR angiography revealed no abnormal findings. The main venous sinuses were confirmed to be patent by 3 dimensional reconstructions of MRI with gadolinium (Fig. 2). Chest X-ray did not reveal any abnormal lesions, and the results of serum examination for infectious diseases, including human immunodeficiency virus (HIV), were negative except for hepatitis B virus surface antigen.

Subsequently, the patient was diagnosed with subacute cerebellar infarction due to arteriosclerosis and was administered glycerol to control the intracranial pressure; however, 1 week after admission, his GCS decreased to 11. Computed tomography confirmed worsened cerebellar edema and hydrocephalus. External and internal decompression surgery were performed to control the intracranial pressure (Fig. 3a, d). A section of the swollen cerebellar hemisphere was removed and submitted as a surgical specimen. Additionally, external continuous ventricular drainage was performed to control hydrocephalus. Lumbar puncture to collect cerebral spinal fluid (CSF) was not performed until this time because of the risk of cerebral herniation. CSF from continuous ventricular drainage demonstrated mild inflammation (cell count, $36 / \mathrm{mm}^{3}$; protein, $16 \mathrm{mg} / \mathrm{dl}$; glucose, $113 \mathrm{mg} / \mathrm{dl})$. C. neoformans was detected in CSF as well as in the surgical specimen of the cerebellum.

Histopathologic examination of the surgical specimen revealed strong hyperplasia of the arachnoid mater (Fig. $4 \mathrm{a})$. Fungi were mainly localized in the subarachnoid space and rarely in the parenchyma (Fig. 4c). Lymphocytes and multinucleated giant cells forming granulomata invaded the arachnoid and subarachnoid spaces

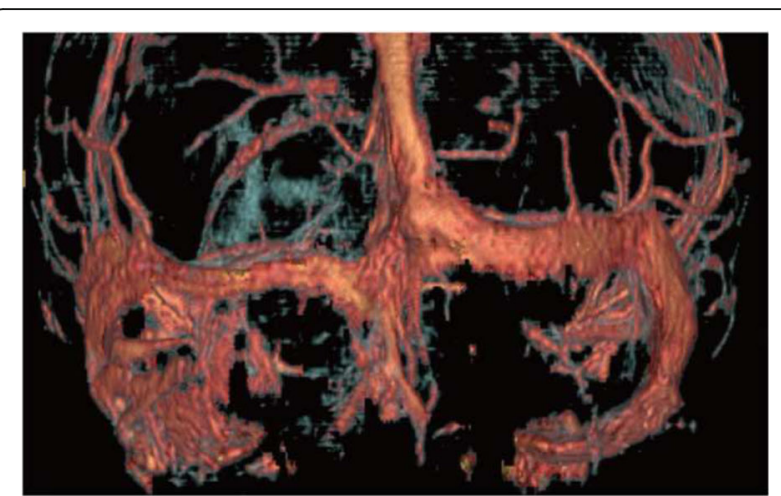

Fig. 2 Reconstruction of gadolinium enhanced magnetic resonance imaging performed on admission. Although sinus venous thrombosis was suspected to be the cause of the observed cerebellar infarction with edema, the main venous sinuses were confirmed to be patent

and pia with heavy fibrosis (Fig. 4b-f). Small arteries were occasionally observed to be occluded with internal endothelial proliferation. While there were arteries in the sample, veins were rarely observed in the subarachnoid space (Fig. $4 \mathrm{~d}$ ). In addition, venules in the parenchyma were frequently observed to be congested. The patient was diagnosed with granulomatous meningitis due to $C$. neoformans and was immediately treated using liposomal amphotericin B and fluconazole; however, the ischemic lesion of the cerebellum continued to bilaterally worsen along with worsening perilesional edema (Fig. 3b-f). The patient's course subsequently deteriorated. He developed renal failure and ultimately died 25 days after admission.

Autopsy confirmed that the pathological changes were confined to the central nervous system and predominantly localized at the surface of the cerebellar hemisphere. Fungal bodies were widely spread along the surface and bilaterally into the deep sulcus of the cerebellum (Fig. 4g). Few fungi were observed in the supratentorial and intraparenchymal lesions. The lesion in the arachnoid mater of the cerebellum was roughly the same as that in the surgical specimen although it was more deeply spread into the peripheral

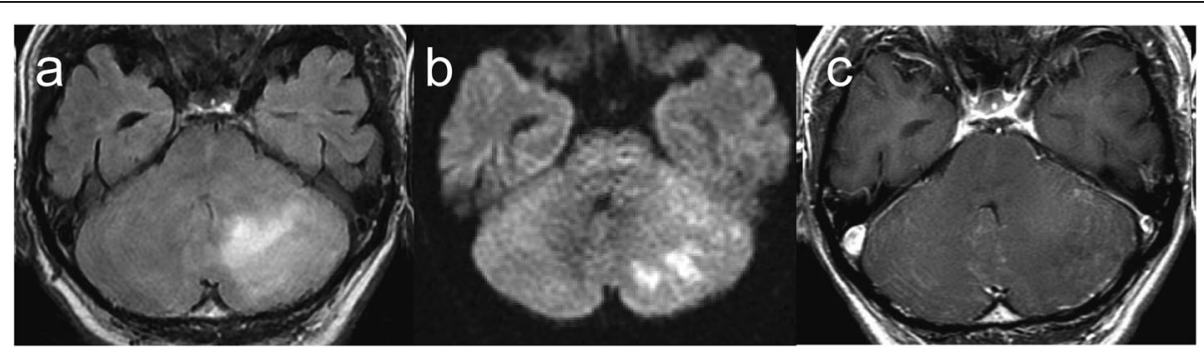

Fig. 1 Brain magnetic resonance imaging performed on admission. The findings on diffusion weighted imaging (DWI) mimicked arterial infarction of the posterior inferior cerebellar artery accompanying strong perilesional edema with almost no enhancement of the lesion. a Fluid-attenuated inversion recovery (FLAIR) image on admission. b DWI. c T1-weighted imaging with gadolinium enhancement 

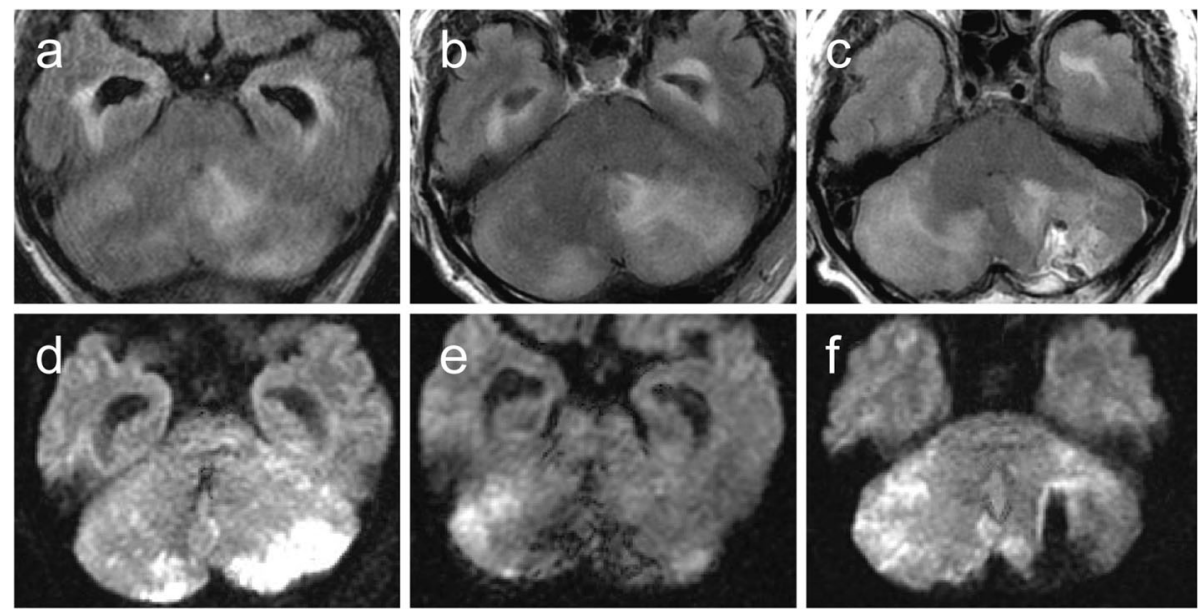

Fig. 3 Progression of cerebellar infarction in Cryptococcal meningoencephalitis. a, d FLAIR (a) and DWI (d) performed following internal and external decompression 10 days after admission. The ischemic lesion progressed to the contralateral cerebellar hemisphere with peripheral edema and ventricular dilatation unresponsive to external continuous ventricular drainage. b, e. FLAIR (b) and DWI (e) performed 17 days after admission. The ischemic and edematous lesion progressed from dorsal to ventral. c, f FLAIR (c) and DWI (f) performed 23 days after admission, 3 days before the patient died. The cerebellum had completely swollen, and tight cisterns were observed indicating brain herniation

sulcus and the granulomatous inflammation was not as severe (Fig. 4h).

\section{Discussion}

We observed two key results in this study: CM can result in massive infarction by subarachnoid small vein occlusion and an atypical infarction on MRI can be observed.

Firstly, C. neoformans can induce extensive subarachnoid small vein occlusion due to inflammatory fibrosis leading to poor outcomes. Generally, C. neoformans tends to hematogenously spread to the brain along the surface, thus inducing meningitis. When the fungi reach the perivascular space of perforating arteries, they begin to invade the perivascular space toward the deeper parts of the brain, simultaneously presenting cerebral infarction mimicking lacunar infarction; however, the mechanism of this process has not been described previously $[4,5]$. In contrast, the most unprecedented finding of our case was that the fungi were present not only at the superficial subarachnoid space of the cerebellum but also diffusely spread and deeply within the sulcus (Fig. 4b-h). Peripheral subarachnoid spaces were thickened with increased internal elastic fibers (Fig. 4b-h). While small arteries showed proliferation of endothelial cells (Fig. 4d, f arrows), veins were rarely observed, which indicates that they had been compressed. Meanwhile, venules from the parenchyma were frequently observed to be congested at the entry of the fibroid subarachnoid space (Fig. 4h, arrow head). It can be speculated that the wide spread fungal involvement triggered granulomatous inflammation, which subsequently induced fibrosis. The compression of small veins in the subarachnoid space may have been due to thickened fibroid tissue, thus blocking the venous return from the parenchyma. This may consequently lead to venous infarction with accompanying massive edema and mass effect. Although the broadly scattered fungi may underlie this pathophysiology, it was unclear how the fungi could widely invade the subarachnoid space and deeply invade the cerebellum.

Secondly, the temporal change of the infarction on MRI was unusual compared with that in the previous cases of CM. Cerebral infarctions are observed in 4$32 \%$ of patients with CM on MRI, typically lacunar at the basal ganglia [6-9]. In our case, multiple infarctions with variable phases in the left cerebellar hemisphere mimicked arterial infarctions of the posterior inferior cerebellar artery with edema on admission MRI (Fig. 1a, b). Subsequently, the ischemic lesion progressed independent of the territory of the artery (Fig. 3b-f). The lesion gradually spread from the left to the right cerebellar hemisphere and from the surface to deeper parts of the cerebellum (Fig. 3b-d). The slow progression of the initial infarction at the territory of posterior inferior cerebellar artery might may be a result of small arterial occlusion due to endothelial proliferation (Fig. 1, Fig. 4d, e), and this condition may have partly contributed to the edema. However, massive edema could not be explained by arterial occlusion alone. Together with the fact that the main venous sinuses were patent (Fig. 2) and that venules were widely congested, the massive cerebellar edema may be predominantly due to occlusion of small veins at the subarachnoid space rather than due to occlusion of large veins such as sinuses [10]. The findings on autopsy indicated that the unique 

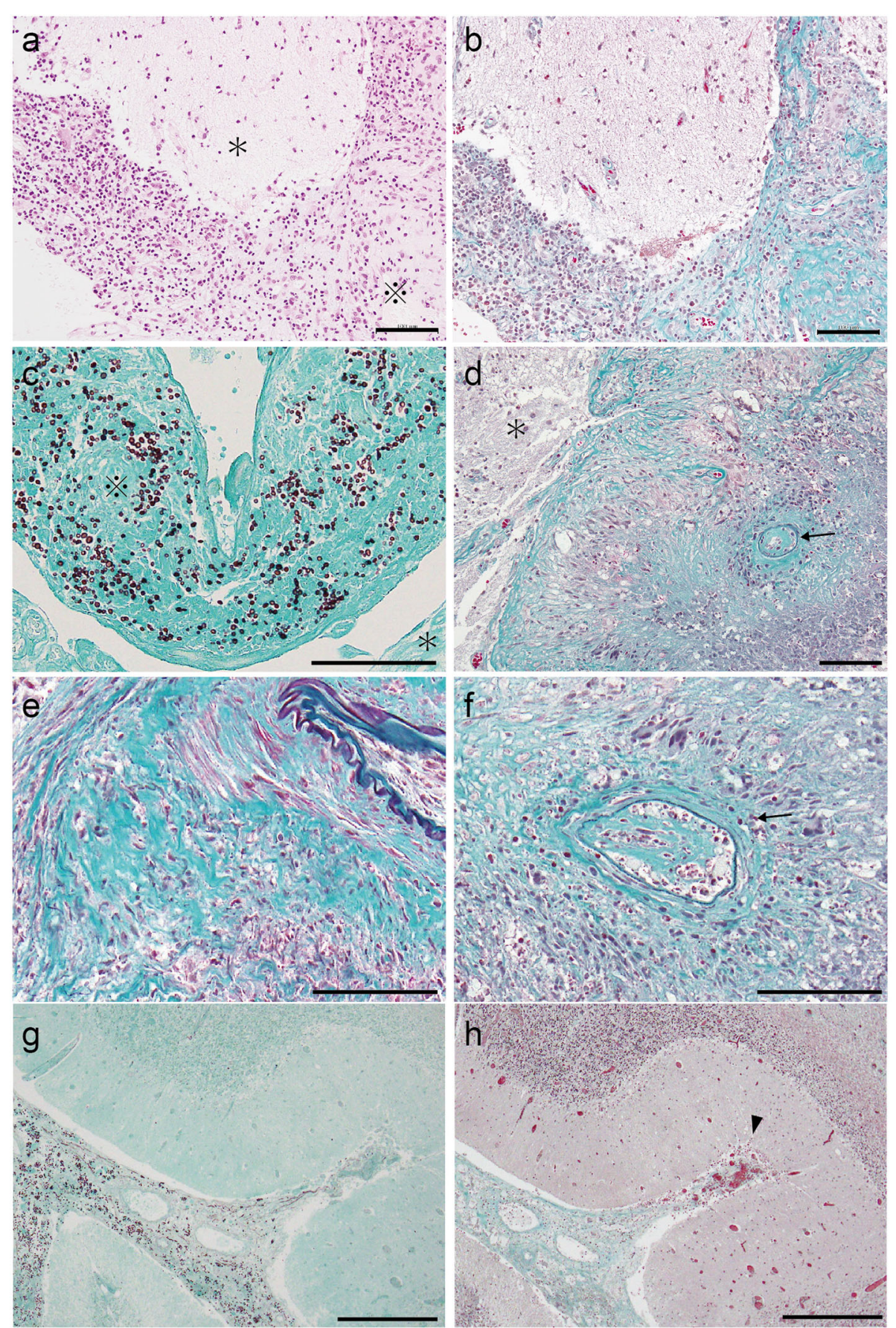

Fig. 4 Pathological findings of the cerebellum specimen. a Hematoxylin and Eosin staining of the left cerebellar hemisphere from the surgical specimen. The subarachnoid space was heavily thickened with inflammatory cells, which is expressed as $※$. The parenchyma is expressed as *. Scale bar: $100 \mu \mathrm{m}$. b Elastica-Masson staining at the same location as in (a). Fibrosis was widely observed in the subarachnoid space, which appears green. Scale bar: $100 \mu \mathrm{m}$. c Grocott staining of left cerebellar hemisphere from the surgical specimen. Fungi were observed in the subarachnoid space (※) and few fungi were observed inside the parenchyma (*). Scale bar: $100 \mu \mathrm{m}$. d, e, $\mathbf{f}$ Elastica-Masson staining of the left cerebellar hemisphere from the surgical specimen. Strong and diffuse fibrosis was observed in the subarachnoid space (e). Proliferation of endothelial cells was observed inside the inner cavity of small arteries (arrows in $\mathbf{d}$, $\mathbf{f}$ ), which implies the slow progression of arterial occlusion. Veins were rarely observed in the subarachnoid space, which indicates that they were compressed and occluded. The parenchyma is expressed as *. Scale bar: $100 \mu \mathrm{m}$. $\mathbf{g}$ Grocott staining of the deep sulcus in the left cerebellar hemisphere from the autopsy specimen. The subarachnoid space was filled with fungi not only at the surface of the cerebellar hemisphere but also in the deep sulcus. Scale bar: $500 \mu \mathrm{m}$. $\mathbf{h}$ Elastica-Masson staining at the same location as in (e). Fibrosis and thickening of the subarachnoid space was observed even in the deep sulcus of the cerebellar hemisphere. Venules from the parenchyma were frequently observed to be congested at the entry to the fibrinous subarachnoid space (arrow head). Scale bar: $500 \mu \mathrm{m}$ 
progression of the ischemic and edematous lesion on MRI reflected the invasion of the fungi and aggressive fibrosis.

Some cases of CM are known to present with fulminant intracranial pressure elevation, which leads to brain herniation and death [11-16]. Zhu reported that cerebral herniation was observed in $30(19.5 \%)$ of 154 nonHIV patients with CM reviewed in their study. In 30 patients who presented with cerebral herniation, 21 died within a year of the diagnosis and 11 within a week, 10 of whom died due to cerebral herniation. They also found that cerebral herniation was one of the crucial and independent factors for poor prognosis in patients with CM [11]. Thus, although cerebral herniation is known to be the most common cause of death in patients with $\mathrm{CM}$, the mechanisms of herniation are not well described. Hydrocephalus due to impaired absorption or obstruction of CSF may be one of the primary reasons for cerebral herniation [12, 17]. The temporal change of the MRI and the findings from autopsy indicate that subarachnoid vein occlusion is a potential mechanism for massive brain edema and an aggressive clinical course in CM. When the fungi broadly and deeply spread into the sulcus of the cerebellum, inflammatory fibrosis widely occurs and might lead to venule congestion and, ultimately, to life threatening massive brain edema. Infarction in lesions, independent of the vascular territory, may be a sign of the progression of fibrosis of the subarachnoid space and decreased compliance of the brain. To our knowledge, this is the first case demonstrating the clinical course and pathological findings of subarachnoid vein occlusion caused by $C$. neoformans. Further reports are necessary to characterize the time course of $C$. neoformans in the central nervous system.

\section{Conclusions}

In conclusion, we suggest that $\mathrm{CM}$ induces subarachnoid small vein occlusion via inflammatory fibrosis, which leads to an aggressive and fatal clinical course. In this case, the temporal change in perifocal edema and ischemic lesions suggested an etiology different from that of a simple arterial infarction. Hence, CM should be considered as a cause of progressive cerebral infarction even in the absence of prior HIV.

\section{Abbreviations}

CM: Cryptococcal meningoencephalitis; DWI: Diffusion weighted image; FLAIR: Fluid-attenuated inversion recovery; GCS: Glasgow coma scale; MRI: Magnetic resonance imaging

\section{Acknowledgments}

The authors thank Enago (http://www.enago.com) for the English language review.
Funding

None of the authors have received any financial assistance for this manuscript.

\section{Availability of data and materials}

The datasets generated during the present study are available from the corresponding author upon reasonable request.

\section{Authors' contributions}

YS, SO, and HA collected the data, designed, and wrote the manuscript. TT wrote and gave the final approval of the manuscript. HA and SO were involved with the clinical care of the patient. TO preformed the histologica examination and interpreted the data. All authors read and approved the final manuscript.

Ethics approval and consent to participate

Not applicable.

\section{Consent for publication}

Written informed consent was obtained from the patient's wife for publication of this case report and any accompanying images. A copy of the written consent is available for review by the Editor of this journal.

\section{Competing interests}

The authors declare that they have no competing interests.

\section{Publisher's Note}

Springer Nature remains neutral with regard to jurisdictional claims in published maps and institutional affiliations.

\section{Author details}

'Department of Neurosurgery, South Miyagi Medical Center, 38-1 Azanishi, Ogawara-machi, Shibata-gun, Miyagi 989-1253, Japan. ²Department of Neurosurgery, Tohoku University Graduate School of Medicine, Sendai, Japan. ${ }^{3}$ Department of Pathology, South Miyagi Medical Center, Shibata-gun, Miyagi, Japan.

Received: 3 April 2017 Accepted: 31 July 2017

Published online: 09 August 2017

\section{References}

1. Williamson PR, Jarvis JN, Panackal AA, Fisher MC, Molloy SF, Loyse A, et al. Cryptococcal meningitis: epidemiology, immunology, diagnosis and therapy. Nat Rev Neurol. 2017:13(1):13-24

2. Ye S, Yang C-d. Central nervous system infections in the systemic vasculitides. Curr Opin Neurol. 2015:4(1):96-104.

3. Sarkis RA, Mays M, Isada C, Ahmed M. MRI findings in cryptococcal meningitis of the non-HIV population. Neurologist. 2015;19(2):40-5.

4. Klock C, Cerski M, Goldani LZ. Histopathological aspects of neurocryptococcosis in HIV-infected patients: autopsy report of 45 patients. Int J Surg Pathol. 2009;17(6):444-8.

5. Lee SC, Dickson DW, Casadevall A. Pathology of cryptococcal meningoencephalitis: analysis of 27 patients with pathogenetic implications. Hum Pathol. 1996;27(8):839-47

6. Tien RD, Chu PK, Hesselink JR, Duberg A, Wiley C. Intracranial cryptococcosis in immunocompromised patients: CT and MR findings in 29 cases. AJNR Am J Neuroradiol. 1991:12(2):283-9.

7. Lan SH, Chang WN, Lu CH, Lui CC, Chang HW. Cerebral infarction in chronic meningitis: a comparison of tuberculous meningitis and cryptococcal meningitis. QJM. 2001;94(5):247-53

8. Chen S-F, Lu C-H, Lui C-C, Huang C-R, Chuang Y-C, Tan T-Y, et al. Acute/ subacute cerebral infarction (ASCI) in HIV-negative adults with cryptococcal meningoencephalitis (CM): a MRI-based follow-up study and a clinical comparison to HIV-negative CM adults without ASCI. BMC Neurol. 2011; 11(1):12.

9. Xia S, Li X, Shi Y, Liu J, Zhang M, Gu T, et al. A retrospective cohort study of lesion distribution of HIV-1 infection patients with cryptococcal meningoencephalitis on MRl: correlation with immunity and immune reconstitution. Medicine (Baltimore). 2016;95(6):e2654. 
10. Senadim S, Alpaydin BS, Tekin GB, Dedei DM, Kantaroglu E, Ozturk O, et al. A rare cause of cerebral venous thrombosis: cryptococcal meningoencephalitis. Neurol Sci. 2016;37:1145-8.

11. Zhu L-P, Wu J-Q, Xu B, Ou X-T, Zhang Q-Q, Weng X-H. Cryptococcal meningitis in non-HIV-infected patients in a Chinese tertiary care hospital, 1997-2007. Med Mycol. 2010;48(4):570-9.

12. Orsini J, Blaak C, Mahmoud D, Young-Gwang J. Massive cerebral edema resulting in brain death as a complication of Cryptococcus neoformans meningitis. J Community Hosp Intern Med Perspect. 2015;5(1):26098.

13. Hamilton RL, Lane H, Browne L, Delanty N, Neill SO, Thornton J, et al. 40year-old man with headaches and dyspnea. Brain Pathol. 2005;15(1):89-90.

14. Teo YK. Cryptococcal meningoencephalitis with fulminant intracranial hypertension: An unexpected case of brain death. Singap Med J. 2010;51: e133-6.

15. Bromilow J, Corcoran T. Cryptococcus gattii infection causing fulminant intracranial hypertension. Br J Anaesth. 2007;99:528-31.

16. Terada T. Cryptococcosis in the central nervous system in a 36-year-old Japanese man: an autopsy study. Tohoku J Exp Med. 2010;222(1):33-7.

17. Loyse A, Wainwright H, Jarvis JN, Bicanic T, Rebe K, Meintjes G, et al. Histopathology of the arachnoid granulations and brain in HIV-associated cryptococcal meningitis: correlation with cerebrospinal fluid pressure. AIDS. 2010:24:405-10.

\section{Submit your next manuscript to BioMed Central} and we will help you at every step:

- We accept pre-submission inquiries

- Our selector tool helps you to find the most relevant journal

- We provide round the clock customer support

- Convenient online submission

- Thorough peer review

- Inclusion in PubMed and all major indexing services

- Maximum visibility for your research

Submit your manuscript at www.biomedcentral.com/submit 